\title{
SOME THEOREMS ON TENSOR DIFFERENTIAL INVARIANTS
}

\author{
BY JACK LEVINE
}

1. Introduction. In the theory of algebraic invariants there is a theorem which states that if an absolute invariant be written as the quotient of two relatively prime polynomials, then the numerator and denominator are relative invariants.* If we consider absolute scalar differential invariants of a metric (or affine) space, then it is possible to prove a similar theorem regarding them. In the course of the proof we give a new proof of the fact that in a relation of the form (2) the $\phi$ must be a power of the Jacobian of the coordinate transformation. (In the algebraic theory the $u_{j}^{i}$ are of course constants.) This proof involves the use of the differential equations satisfied by the scalar. $\dagger$ In this proof it is not necessary to restrict $B$ and $\phi$ to be polynomials in their arguments as is done in the usual proof of the corresponding theorem in the invariant theory. It is sufficient to assume that $\phi$ possesses first derivatives with respect to the $u_{j}^{i}$ and that $B(\bar{g})$ is an analytic function of $\epsilon$ in the neighborhood of $\epsilon=0$. We also extend the theorem to the case of tensor differential invariants of the form (5).

2. Scalar Differential Invariants. We consider the differential invariants of a metric space $V_{n}$ with a quadratic form $g_{i j} d x^{i} d x^{j}$. Let

$$
A\left(g_{i j} ; \frac{\partial g_{i j}}{\partial x^{k}} ; \cdots ; \frac{\partial^{p} g_{i j}}{\partial x^{k} \cdots \partial x^{l}}\right)
$$

be an absolute scalar invariant of $V_{n}$ which we take to be rational in its arguments. We can then write $A$ in terms of the $g_{i j}$ and their extensions $g_{i j, k} \ldots l$, and we have

$$
A\left(g_{i j} ; 0 ; g_{i j, k l} ; \cdots\right)=\frac{B\left(g_{i j} ; 0 ; g_{i j, k l} ; \cdots\right)}{C\left(g_{i j} ; 0 ; g_{i j, k l} ; \cdots\right)},
$$

\footnotetext{
* See, for example, H. W. Turnbull, The Theory of Determinants, Matrices, and Invariants, p. 277.

$\dagger$ T. Y. Thomas and A. D. Michal, Differential invariants of relative quadratic differential forms, Annals of Mathematics, vol. 28 (1927), p. 679.
} 
where $B$ and $C$ are polynomials in the $g_{i j}$ and their extensions. We may assume that $B$ and $C$ have no common factor. Now

$$
\frac{B\left(g_{i j} ; \cdots\right)}{C\left(g_{i j} ; \cdots\right)}=\frac{B\left(\bar{g}_{i j} ; \cdots\right)}{C\left(\bar{g}_{i j} ; \cdots\right)}
$$

under an arbitrary coordinate transformation $x \rightarrow \bar{x}$, the barred $g$ 's being the $g$ 's in the $(\bar{x})$ coordinate system. It is easily shown we must have*

$$
\begin{aligned}
& B\left(\bar{g}_{i j} ; \cdots\right)=\phi\left(u_{b}^{a}\right) B\left(g_{i j} ; \cdots\right), \\
& C\left(\bar{g}_{i j} ; \cdots\right)=\phi\left(u_{b}^{a}\right) C\left(g_{i j} ; \cdots\right),
\end{aligned}
$$

where $u_{b}^{a}=\partial x^{a} / \partial \bar{x}^{b}$ and $\phi$ is a polynomial in the $u^{\prime}$ s. We now prove $\phi$ is a power of $|\partial x / \partial \bar{x}|$, thus showing $B$ and $C$ are relative scalars.

Write (2) in the form

$$
B(\bar{g}) \phi^{-1}=B(g),
$$

and consider the infinitesimal transformation

$$
x^{i}=\bar{x}^{i}+\epsilon \xi^{i}(\bar{x}) .
$$

We have

$$
\left(\frac{d \phi^{-1}}{d \epsilon}\right)_{\epsilon=0}=-\left(\frac{\partial \phi}{\partial u_{j}^{i}}\right)_{\epsilon=0}\left(\frac{d u_{j}^{i}}{d \epsilon}\right)_{\epsilon=0}=-\left(\frac{\partial \phi}{\partial u_{j}^{i}}\right)_{\epsilon=0} \frac{\partial \xi^{i}(x)}{\partial x^{j}} .
$$

As $\phi$ is a polynomial, so also is $\partial \phi / \partial u_{j}^{i}$, and on evaluating this last expression at $\epsilon=0$, we obtain a set of constants $k_{i}^{j}$, so that

$$
\left(\frac{d \phi^{-1}}{d \epsilon}\right)_{\epsilon=0}=-k_{i}^{j} \frac{\partial \xi^{i}}{\partial x^{j}} .
$$

Proceeding then as in the paper by Thomas and Michal, page 679 , we obtain the differential equations satisfied by $B$ in the form

$$
X_{s}^{t}(p) B=k_{s}^{t} B .
$$

Now for any function $G$ we have $\dagger$

$$
\left(X_{s}^{t}, X_{m}^{l}\right) G=\delta_{m}^{t} X_{s}^{l} G-\delta_{s}^{l} X_{m}^{t} G,
$$

${ }^{*}$ H. W. Turnbull, loc. cit.

$\dagger$ Thomas and Michal, loc. cit., p. 663. 
and, in particular, for $B$ we would have

$$
\left(X_{s}^{t}, X_{m}^{l}\right) B=\delta_{m}^{t} X_{s}^{l} B-\delta_{s}^{l} X_{m}^{t} B=B\left(\delta_{m}^{l} k_{s}^{l}-\delta_{s}^{l} k_{m}^{t}\right) .
$$

But also

$$
\left(X_{s}^{t}, X_{m}^{l}\right) B=X_{s}^{t}\left(k_{m}^{l} B\right)-X_{m}^{l}\left(k_{s}^{t} B\right)=B\left(k_{m}^{l} k_{s}^{t}-k_{s}^{t} k_{m}^{l}\right)=0,
$$

so that

$$
\delta_{m}^{t} k_{s}^{l}-\delta_{s}^{l} k_{m}^{t}=0
$$

from which follows $k_{m}^{l}=k \delta_{m}^{l}$, where $k$ is a constant. Substituting in (4) we find that $B$ is a relative scalar of weight $k$ and* $\phi=|\partial x / \partial \bar{x}|{ }^{k}$. Similar results hold for $C$ also. As stated in $\S 1$ we can prove a generalization of this result which we state as follows.

THEOREM 1. Given a function

$$
B\left(g_{i j} ; \frac{\partial g_{i j}}{\partial x^{k}} ; \cdots ; \frac{\partial^{p} g_{i j}}{\partial x^{k} \cdots \partial x^{l}}\right),
$$

with the law of transformation

$$
B\left(\bar{g}_{i j} ; \cdots ; \frac{\partial^{p} \bar{g}_{i j}}{\partial \bar{x}^{k} \cdots \partial \bar{x}^{l}}\right)=\phi\left(u_{b}^{a}\right) B\left(g_{i j} ; \cdots ; \frac{\partial^{p} g_{i j}}{\partial x^{k} \cdots \partial x^{l}}\right),
$$

where $\phi$ possesses first derivatives in the $u$ 's and $B(\bar{g})$ is analytic in the neighborhood of $\epsilon=0$. Then $\phi$ is a power of the Jacobian and $B$ is a relative scalar differential invariant.

3. Tensor Differential Invariants. Consider the absolute tensor differential invariant with components of the form

$$
T_{i \cdots j}^{a \cdots b}=\frac{U_{i \cdots j}^{a \cdots b}\left(g_{k l} ; g_{k l, m q} ; \cdots\right)}{D\left(g_{k l} ; \cdots\right)},
$$

where the $U$ 's and $D$ are polynomials (with no common factor) in their arguments. Corresponding to (1) in the scalar case we have

$$
\frac{U_{i \cdots j}^{a \cdots b}(g) u_{k}^{i} \cdots u_{l}^{j}}{U_{k \cdots l}^{\prime m \cdots p}(g, u) u_{m}^{a} \cdots u_{p}^{b}}=\frac{D(g)}{D^{\prime}(g, u)}=\frac{Q(g, u)}{P(g, u)}
$$

* Thomas and Michal, loc. cit. 
( $P, Q$ having no common factor), where the primed $U$ 's and $D$ represent the result of replacing the barred $g$ 's by their values in terms of the $g$ 's and $u$ 's in the expressions $U \cdots(\bar{g})$ and $D(\bar{g})$. From (6) we see that $Q$ is a factor of $D$ and of $U_{i \ldots j}^{a} \ldots b i u_{k}^{i} \cdots u_{l}^{j}$, and hence must be a function $Q^{\prime}(g)$, so that

$$
\begin{aligned}
U_{i \cdots j}^{a \cdots b}(g) u_{k}^{i} \cdots u_{l}^{j} & =Q^{\prime}(g) V_{k \cdots l}^{a \cdots b}(g, u), \\
D(g) & =Q^{\prime}(g) R(g) .
\end{aligned}
$$

In (7) put $u_{j}^{i}=\delta_{j}^{i}$; then

$$
U_{k \cdots l}^{a \cdots b}(g)=Q^{\prime}(g) V_{k \cdots l}^{a \cdots b}(g, \delta) .
$$

Hence $Q^{\prime}(g)=$ const., since $D$ and the $U$ 's have no common factor. Since $D(g)$ and $D^{\prime}(g, u)$ are of the same degree in the $g$ 's, it then follows from (6) that $P(g, u)=P^{\prime}(u)$, so that

$$
D(\bar{g})=\phi\left(u_{j}^{i}\right) D(g) .
$$

Hence as proved in the previous section for $B$, we have shown that $D$ is a relative scalar of weight $k$, and therefore $U_{i}^{a} \ldots j$ are the components of a relative tensor of weight $k$. We can also prove the following theorem.

THEOREM 2. If the set of quantities

$$
T_{a \cdots b}^{i \cdots j}\left(g_{k l} ; \cdots ; \frac{\partial^{p} g_{k l}}{\partial x^{m} \cdots \partial x^{r}}\right)
$$

have the transformation law

$$
T_{v \cdots w}^{s \cdots t}\left(\bar{g}_{k l} ; \cdots\right) u_{s}^{i} \cdots u_{t}^{j}=\phi\left(u_{e}^{d}\right) T_{a \cdots b}^{i \cdots i}\left(g_{k l} ; \cdots\right) u_{v}^{a} \cdots u_{w}^{b},
$$

then $\phi$ is a power of $|\partial x / \partial \bar{x}|$ and the T's are components of a relative tensor invariant, it being assumed that $\phi$ possesses first derivatives in the $u^{\prime}$ s, and $T(\bar{g})$ are analytic in the neighborhood of $\epsilon=0$.

The proof is similar to that used for $B$ of the previous section. Similar results to those obtained for metric scalar and tensor differential invariants hold for affine invariants.

Princeton University 